\title{
Bohmian Photonics for Independent Control of the Phase and Amplitude of Waves
}

\author{
Sunkyu Yu, Xianji Piao, and Namkyoo Park ${ }^{*}$ \\ Photonic Systems Laboratory, Department of Electrical and Computer Engineering, \\ Seoul National University, Seoul 08826, Korea
}

(Received 6 December 2017; published 9 May 2018)

\begin{abstract}
The de Broglie-Bohm theory is one of the nonstandard interpretations of quantum phenomena that focuses on reintroducing definite positions of particles, in contrast to the indeterminism of the Copenhagen interpretation. In spite of intense debate on its measurement and nonlocality, the de Broglie-Bohm theory based on the reformulation of the Schrödinger equation allows for the description of quantum phenomena as deterministic trajectories embodied in the modified Hamilton-Jacobi mechanics. Here, we apply the Bohmian reformulation to Maxwell's equations to achieve the independent manipulation of optical phase evolution and energy confinement. After establishing the deterministic design method based on the Bohmian approach, we investigate the condition of optical materials enabling scattering-free light with bounded or random phase evolutions. We also demonstrate a unique form of optical confinement and annihilation that preserves the phase information of incident light. Our separate tailoring of wave information extends the notion and range of artificial materials.
\end{abstract}

DOI: 10.1103/PhysRevLett.120.193902

The de Broglie-Bohm theory [1], also called Bohmian mechanics, suggests an alternative interpretation of quantum mechanics for the description of individual events in statistical quantum phenomena. Compared to the Copenhagen interpretation, which emphasizes the indeterministic nature of quantum mechanics [2], the de BroglieBohm approach, constructed upon the reformulation of the Schrödinger equation [1,3], attempts to reintroduce a definite position of each particle that is guided by a pilot wave [4]. This causal view for "point particles" enables the understanding of quantum phenomena in a classical-like sense [3], though there has been considerable debate [5-10] regarding the measurement and nonlocality of the Bohmian approach, such as surreal trajectories in a Welcher-Weg measurement $[5,10]$. To our knowledge, at this stage, the de Broglie-Bohm theory does not contradict the orthodox Copenhagen interpretation and quantum-mechanical experiments. Recent achievements are discussed in [11].

The heart of the Bohmian formulation is in the application of the polar-form wave function $\psi=R e^{i(S / \hbar)}$ to the Schrödinger equation $i \hbar \partial_{t} \psi=-\left(\hbar^{2} / 2 m\right) \nabla^{2} \psi+V \psi$ to separate the real and imaginary parts of this complex-valued equation [3]. The resulting quantum Hamilton-Jacobi (QHJ) equation $\partial_{t} S+(\nabla S)^{2} / 2 m-\left(\hbar^{2} / 2 m\right) \nabla^{2} R / R+V=0$ and the continuity equation $\partial_{t} R^{2}+\nabla \cdot\left(R^{2} \nabla S / m\right)=0$ identify

Published by the American Physical Society under the terms of the Creative Commons Attribution 4.0 International license. Further distribution of this work must maintain attribution to the author(s) and the published article's title, journal citation, and DOI. an individual particle trajectory in the form of Newton's second law, in which the exerted force is represented by two parts: the classical force $-\nabla V$ and the quantum force $\left(\hbar^{2} / 2 m\right) \nabla\left(\nabla^{2} R / R\right)$. The annihilation of the quantum force from $\hbar \rightarrow 0$ then approximates quantum phenomena by Newtonian mechanics.

In this Letter, we show that the Bohmian formulation of Maxwell's wave equation provides a new perspective on optical materials for the independent tailoring of amplitude and phase information of light, which has not been reported so far. By deriving the optical counterpart of the QHJ equation, we demonstrate that optical potentials can be understood as the sum of the classical-like part for the phase evolution and the quantumlike part for the energy confinement. In this vein, as a first example, we realize a quantum-mechanically-free potential [3] in an optical platform, to derive a new class of constant-intensity waves $[12,13]$ that have freely designed phase distributions with phase trapping or randomization functions. Anomalous confinement or annihilation of energy that perfectly preserves the phase information of light will also be demonstrated with the construction of a scattering-free quantumlike potential. These results not only pave the way for the independent manipulation of wave quantities but also deepen the understanding of artificial materials, such as the near-zero regime [14] in optical material parameters.

To explore the consequences of the Bohmian approach in optics, we consider a monochromatic wave of frequency $\omega$ propagating in the $x-y$ plane within a medium that has a permittivity $\varepsilon(x, y)=\varepsilon_{r}(x, y)+i \varepsilon_{i}(x, y)$. For the transverse electric mode (electric field along the $z$ axis), the field evolution obeys the Helmholtz wave equation 
$\nabla^{2} E_{z}+V(x, y) E_{z}=0$, where $V=k_{0}^{2} \varepsilon(x, y)$ and $k_{0}=\omega / c$. We then express the field profile in polar form [3] as $E_{z}(x, y)=R(x, y) e^{i S(x, y)}$, where $R$ is a real amplitude function that is by definition non-negative, and $S$ is a real phase function that determines the instantaneous wave vector $\mathbf{k}(x, y)=\nabla S$, which corresponds to the guidance equation [3] of light. Applying this polar-form electric field to the Helmholtz equation allows the separation of the real and imaginary parts of the wave equation to yield the following representations of the complex optical potential $V=V_{r}(x, y)+i V_{i}(x, y)$ (Supplemental Material Note S1 in [15])

$$
\begin{aligned}
& V_{r}(x, y)=|\nabla S|^{2}-\frac{\nabla^{2} R}{R}, \\
& V_{i}(x, y)=-\nabla^{2} S-2 \frac{\nabla R}{R} \cdot \nabla S .
\end{aligned}
$$

It is noted that Eq. (1) corresponds to the optical counterpart of the stationary $\left(\partial_{t} \rightarrow 0\right)$ QHJ equation [3]. Similar to the QHJ equation, the real part of the optical potential $V_{r}=k_{0}^{2} \varepsilon_{r}$ [Fig. 1(a)] can always be decomposed into two parts, for each wave information $S$ and $R$ : the "classical" Hamilton-Jacobi term $V_{\text {classical }}=|\nabla S|^{2}$ [Fig. 1(b)] and the "quantum potential" counterpart [Fig. 1(c)] $V_{\text {quantum }}=-\nabla^{2} R / R$. Each potential separately governs the spatial phase evolution with $S(x, y)$ [Fig. 1(d)] and the optical confinement with $R(x, y)$ [Fig. 1(e)], thus constructing the full spatial information of the wave as $E_{z}=R e^{i S}$ [Fig. 1(f)]. Meanwhile, Eq. (2), rewritten as $\nabla \cdot\left(R^{2} \nabla S\right)+k_{0}^{2} \varepsilon_{i}(x, y) R^{2}=0$, is the stationary continuity equation describing the equilibrium between optical sources (or sinks) represented by $\varepsilon_{i}(x, y)$ and the divergence of the power flow $R^{2} \nabla S$.

Critically, applying the Bohmian interpretation of the optical potential allows the independent control of the phase and amplitude distributions of light, analogous to the separation of classical and quantum phenomena in Bohmian mechanics [3]. For the given wave $E_{z}=R e^{i S}$ with targeted spatial functions of $S(x, y)$ and $R(x, y)$ that satisfy the electromagnetic boundary conditions, the real optical potential $V_{r}$ is directly obtained as the sum of $V_{\text {classical }}=|\nabla S|^{2}$ and $V_{\text {quantum }}=-\nabla^{2} R / R$. The imaginary optical potential $V_{i}$ is also determined by the continuity Eq. (2), accomplishing the inverse design of the complex potential $V$ for the given wave $E_{z}=R e^{i S}$.

In light of this Bohmian separation of light-matter interactions in terms of phase and amplitude, we explore a particular example: quantum-mechanically-free [3] potentials of $V_{\text {quantum }}=-\nabla^{2} R / R=0$. Among the general solutions of Laplace's equation $\nabla^{2} R=0$, we consider the constant-intensity (CI) wave [12,13] with $R(x, y)=R_{0}$ to focus on the design of unconventional phase evolution. The complex optical potential for the CI wave then becomes $V_{r}(x, y)=|\nabla S|^{2}$ and $V_{i}(x, y)=-\nabla^{2} S$, which lead to the complex permittivity profile of $\varepsilon(x, y)=\left(|\nabla S|^{2}-i \nabla^{2} S\right) / k_{0}^{2}$.

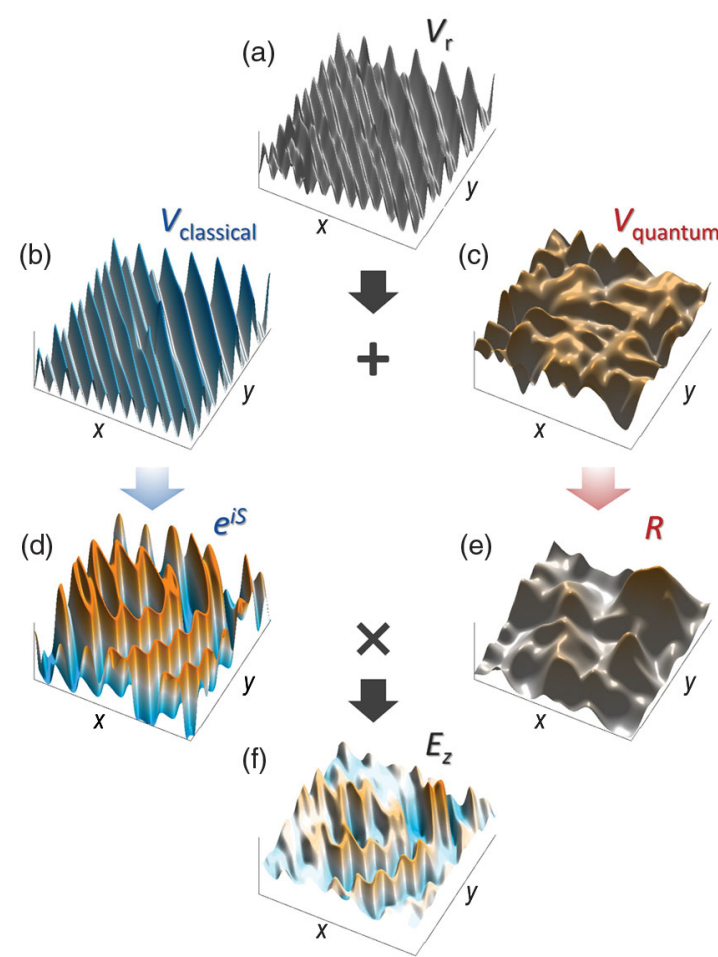

FIG. 1. Bohmian interpretation of an optical potential by separating the classical and quantum potential, each for phase evolution and energy confinement. (a) The real part of the optical potential $V_{r}=k_{0}^{2} \varepsilon_{r} . V_{r}$ is the sum of the (b) classical HamiltonJacobi potential $V_{\text {classical }}=|\nabla S|^{2}$ and (c) quantum potential $V_{\text {quantum }}=-\nabla^{2} R / R$, each governing the (d) phase evolution $e^{i S}$ and (e) amplitude $R$ of the (f) entire light wave $E_{z}=R e^{i S}$.

This expression allows for the alternative classification of conventional "homogeneous" materials to include the following: (i) ordinary material for the linearly varying phase $S(x, y)=k_{x} x+k_{y} y$ with $\varepsilon(x, y)=\left(k_{x}^{2}+k_{y}^{2}\right) / k_{0}^{2}>0$ and (ii) epsilon-near-zero (ENZ) material for the constant phase $S(x, y)=S_{0}$ with $\varepsilon(x, y)=0$. Note that this CI-wave class does not include the case of negative real permittivity because $\operatorname{Re}[\varepsilon]=|\nabla S|^{2} / k_{0}^{2} \geq 0$.

Depending on the form of $S(x, y)$, the CI-wave class can include "inhomogeneous" materials with nonconstant firstorder partial derivatives of $S(x, y)$. Among the many possible designs, we examine spatial phase trapping phenomena (Fig. 2). While conventional materials result in full range phase evolution between 0 and $2 \pi$, the Bohmian design with an appropriate function of $S(x, y)$ enables the "trapping" of the spatial phase in a certain range. For example, taking the one-dimensional (1D) phase evolution as $S(y)=S_{b} \cdot \sin (q y)$, the designed permittivity becomes (Note $S 2$ in [15])

$$
\begin{aligned}
& \varepsilon_{r}(y)=S_{b}^{2}\left(\frac{q}{k_{0}}\right)^{2} \cos ^{2}(q y), \\
& \varepsilon_{i}(y)=S_{b}\left(\frac{q}{k_{0}}\right)^{2} \sin (q y) .
\end{aligned}
$$



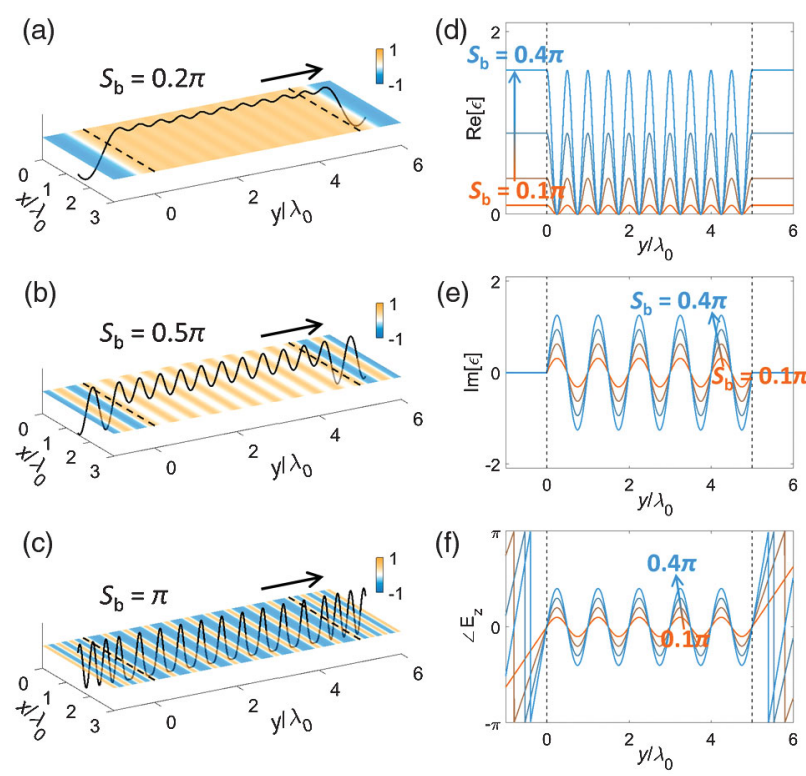

FIG. 2. Bohmian design of spatial phase trapping materials. (a)-(c) Electric field $E_{z}$ evolutions for different values of $S_{b}$ : (a) $S_{b}=0.2 \pi$, (b) $S_{b}=0.5 \pi$, and (c) $S_{b}=\pi$. Arrows in (a)-(c) denote the propagation direction of light. (d) Real and (e) imaginary permittivity landscapes and (f) obtained phase evolutions for $S_{b}=0.1 \pi-0.4 \pi$ with a $0.1 \pi$ interval. The regions between dashed lines denote the designed range according to Eq. (3). The materials in the input and output regions are determined to maintain the continuity of $\varepsilon_{r}(y)$ and $\varepsilon_{i}(y) \cdot q=$ $k_{0}$ for all cases. $\lambda_{0}$ is the free-space wavelength. All of the field evolutions are calculated by the finite element method using COMSOL Multiphysics.

This inhomogeneous complex permittivity allows the "scattering-free" CI wave in which the oscillating phase is bounded in $\left[-S_{b}, S_{b}\right]$ [Figs. 2(a)-2(c) for different values of $S_{b}$ ]. Because the stringent condition of $S_{b}=0$ directly leads to the ENZ material $\left(\varepsilon_{r}=\varepsilon_{i}=0\right)$, the CI-wave class for $S_{b}$-restricted phase trapping materials corresponds to the extension of the phase halt in the ENZ medium. Because the designed phase trapping material can possess a nonzero real part and a zero imaginary part of permittivity at the boundaries [Figs. 2(d) and 2(e)], achieving impedance matching to conventional homogeneous materials while nearly maintaining the properties of ENZ media, i.e., the constant intensity and nearly conserved optical phase, is possible. It is also noted that an exotic phase evolution [here, sinusoidal from the $S(y)$ function, the region between the dashed lines in Fig. 2(f)], in contrast to a linear phase evolution, can be designed, without any scattering due to the CI-wave condition. Although the designed permittivity profiles in Eq. (3) satisfy the parity-time (PT) symmetry [16] with the odd function $S(y)$, it is not a necessary condition for the phase trapping, as shown in Note S3 and Fig. S1 in [15] for the random evolution of the bounded $S(y)$.

The condition of the CI wave can also be extended to two-dimensional (2D) problems. Figure 3 shows an
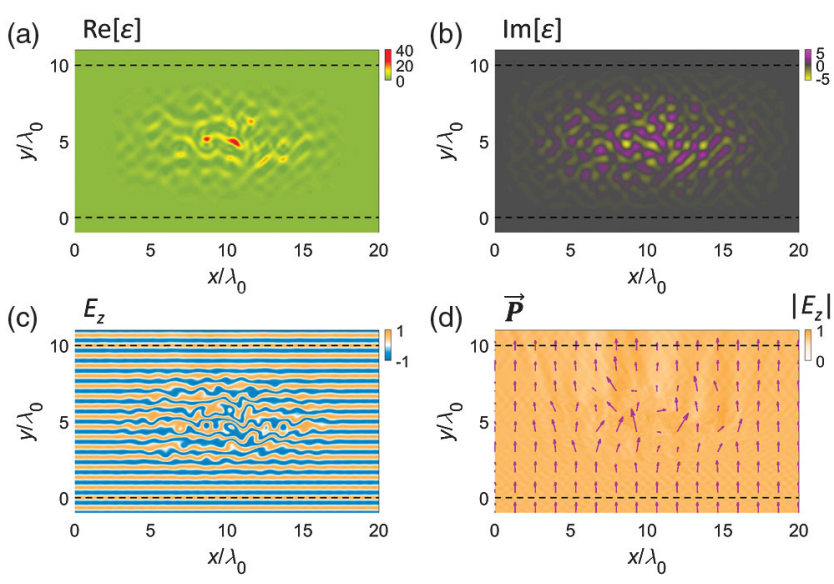

FIG. 3. Bohmian design of the disordered potential for CI-wave propagation. (a) Real and (b) imaginary permittivity distributions and (c) the obtained field evolution. (d) The distribution of the Poynting vector overlaid on the amplitude of the electric field $E_{z}$. The background index is $n_{0}=1.5$. All of the field evolutions are calculated using COMSOL Multiphysics.

example of CI-wave propagation in 2D "disordered" complex potentials. We design the disordered phase function $S(x, y)$ from the plane-wavelike linear phase evolution, with $S(x, y)=n_{0} k_{0} y+2 \pi W(x, y) \Delta S(x, y) /$ $\max \{W(x, y) \Delta S(x, y)\}$ for the background refractive index $n_{0}$, where $\Delta S(x, y)$ is the random perturbation defined in the spectral domain as

$$
\begin{aligned}
\Delta S(x, y)= & u[-1,1] \int_{0}^{k_{0}} \sin \left(q_{x} x+u[-\pi, \pi]\right) d q_{x} \\
& +u[-1,1] \int_{0}^{k_{0}} \sin \left(q_{y} y+u[-\pi, \pi]\right) d q_{y},
\end{aligned}
$$

where $u[a, b]$ is a uniform random number between $a$ and $b$. $W(x, y)$ is the weighting function for the electromagnetic continuity condition of $S(x, y)$ given by

$$
W=\left[1-\cos \left(\frac{2 \pi x}{L_{x}}\right)\right]\left[1-\cos \left(\frac{2 \pi y}{L_{y}}\right)\right],
$$

where $L_{x}$ and $L_{y}$ are the lengths of each side of the rectangle-shaped design area (the regions between the dashed lines in Fig. 3: $0 \leq x \leq 20 \lambda_{0}$ and $0 \leq y \leq 10 \lambda_{0}$ ). From the designed $S(x, y)$, the necessary permittivity distribution is obtained as $\varepsilon(x, y)=\left(|\nabla S|^{2}-i \nabla^{2} S\right) / k_{0}^{2}$ [Figs. 3(a) and 3(b)]. Figure 3(c) shows the spatial field evolution in this disordered medium, showing a random phase in the design area and a perfect plane wave at the input and output regions without any scattering. As shown in the Poynting vector distribution [Fig. 3(d)], this CI-wave propagation in the disordered complex potential is obtained from the modification of the magnetic field while preserving the unity intensity of the electric field. It is also worth mentioning that this Bohmian random potential supporting the CI wave with tunable phase evolution belongs to the 

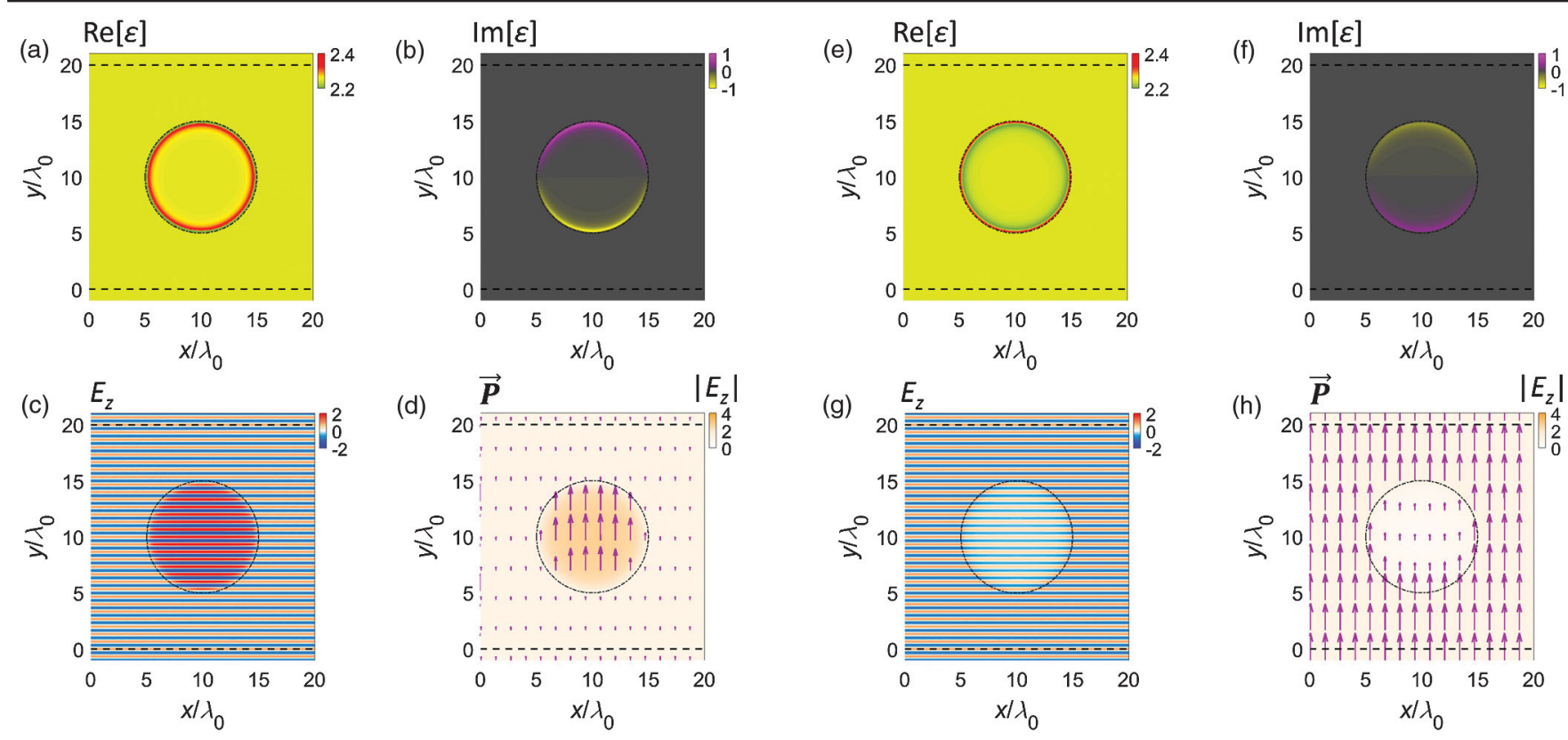

FIG. 4. Phase-conserved control of energy confinement and cancellation, designed by the Bohmian approach. The light energy can be (a)-(d) confined or (e)-(h) annihilated in the designed regions. (a),(e) Real and (b),(f) imaginary permittivity distributions, and (c),(g) obtained field evolutions. (d),(h) The distributions of the Poynting vector overlaid on the amplitude of the electric field $E_{z}$. The confinement and cancellation of optical energy are obtained with (a)-(d) $R_{0}=2$ and (e)-(h) $R_{0}=-0.6$, respectively. $n_{0}=1.5$, $\sigma=5 \lambda_{0}$, and $b=2$ for defining the $2 \mathrm{D}$ bump function. The center of the design area is $\left(x_{0}, y_{0}\right)=\left(10 \lambda_{0}, 10 \lambda_{0}\right)$. All of the field evolutions are calculated using COMSOL Multiphysics.

class of artificial disordered potentials with designed wave profiles [17].

Having demonstrated the phase manipulation with the quantum-mechanically-free case of $V_{\text {quantum }}=0$, we now investigate the utilization of the nonzero quantum potential $V_{\text {quantum }}=-\nabla^{2} R / R \neq 0$, which allows for the realization of exotic energy confinement. The confinement of light is achieved with systems that hinder outgoing waves [18-20], in general, except for a few, such as the use of bound states in the continuum [21,22]. Because all of these methods are inherently based on the scattering of waves, the confinement of light, or, more generally, the modulation of the spatial amplitude distribution $R$, leads to a subsequent, inevitable disturbance to optical phase information $S$. Instead, we apply the Bohmian approach to achieve scattering-free energy confinement, completely preserving the spatial phase information.

We assume a plane-wavelike linear phase evolution $S(x, y)=n_{0} k_{0} y$ in the entire space. The required potential then becomes

$$
\begin{aligned}
& V_{r}(x, y)=n_{0}^{2} k_{0}^{2}-\frac{\nabla^{2} R}{R}, \\
& V_{i}(x, y)=-2 n_{0} k_{0} \frac{\nabla R}{R} \cdot \hat{y} .
\end{aligned}
$$

To satisfy the scattering-free condition for the plane wave excitation, $\nabla R / R$ and $\nabla^{2} R / R$ should be continuous, requiring the $R$ function to be of the $C^{2}$ differentiability class. To fully guarantee the continuity condition, we employ a "bump" function that is smooth, having continuous derivatives of all orders, i.e., of $C^{\infty}$ class

$$
R(x, y)=\left\{\begin{array}{ll}
1+R_{0} \exp \left(-\frac{b^{2} \lambda_{0}^{2}}{\sigma^{2}-r^{2}}\right) & \text { for } r \leq \sigma \\
1 & \text { otherwise }
\end{array},\right.
$$

where $R_{0}$ determines the strength of energy confinement, $\sigma$ defines the confinement area in the polar coordinate representation of $r=\left[\left(x-x_{0}\right)^{2}+\left(y-y_{0}\right)^{2}\right]^{1 / 2}$, and $b$ represents the sharpness of the confinement function. With the calculated permittivity profiles in Figs. 4(a) and 4(b), we show in Figs. 4(c) and 4(d) the clear energy concentration of the light wave inside the confinement region, with perfect conservation of the incident phase information. The negative value of $R_{0}$ in Eq. (7) allows scattering-free energy annihilation inside the designed area, along with an unperturbed phase distribution [Figs. 4(e)-4(h)]. The extension of the result to arbitrary geometries is also shown in Note S4 and Fig. S2 in [15], by utilizing the superposition of bump functions with random distributions. Notably, the spatial profiles of energy confinement and cancellation can be freely designed without any phase distortion.

We have shown that the application of the Bohmian formulation in optics enables the separate understanding of light-matter interactions for optical phase and amplitude, 
each determining the energy and wave vector spatial distribution. From this independent tailoring of wave quantities, we explored two unconventional regimes: a CI wave with spatial phase trapping as an extension of ENZ material and 2D scattering-free disordered materials. The confinement or cancellation of optical energy with perfectly conserved phase information is also achieved, in sharp contrast to traditional scattering-based energy confinement [18-22].

In view of a practical realization, we note that the slower variation of $R(x, y)$ and $S(x, y)$ alleviates the stringent requirements on material values. For example, in Eq. (3), the necessary values of $\varepsilon_{i}$ for the given $S_{b}$ can be significantly decreased with smaller $q$ (e.g., $\max \left|\varepsilon_{i}(y)\right|=$ $4 \times 10^{-3}$ for $q=k_{0} / 20$ and $S_{b}=0.5 \pi$ ), which can be achieved with the subwavelength cell composed of gain dielectric layers [23] and low-loss ENZ structures [14,24]. In the CI-wave and phase-conserved structures, because the real parts of the permittivity are evolved around $n_{0}^{2}$, the necessary material values with gain and loss are accessible with conventional $P T$-symmetric configurations [25,26], with slowly varying $R$ and $S$ [e.g., $\max \left|\varepsilon_{i}(x, y)\right|=$ $2.7 \times 10^{-2}$ for $b=8$, with the same values of $R_{0}, n_{0}$, and $\sigma$ with those in Figs. 4(a)-4(d)].

Compared with transformation optics [27] or the spatial Kramers-Kronig relation [28], our result implies a new design strategy for artificial inhomogeneous media in terms of the independent handling of wave quantities. For example, the inverse design technique for the given phase and amplitude distributions can be directly applied to metamaterial design to obtain largely positive [29], zero [14], or negative [30] real values or imaginary values [25,31-35] of material parameters. The independent control of $S$ and $R$ also enables the intuitive design of antennas and resonators, respectively, in a non-Hermitian regime. The application of the alternative interpretation in quantum mechanics will also provide a new viewpoint in the field of quantum-optical analogy [36].

We acknowledge financial support from the National Research Foundation of Korea (NRF) through the Global Frontier Program (2014M3A6B3063708), the Basic Science Research Program (2016R1A6A3A04009723), and the Korea Research Fellowship Program (2016H1D3A1938069), all funded by the Korean government.

*nkpark@snu.ac.kr

[1] D. Bohm, A suggested interpretation of the quantum theory in terms of "hidden" variables. I, Phys. Rev. 85, 166 (1952).

[2] W. Heisenberg, Physics and Philosophy: The Revolution in Modern Science (George Allen and Unwin, London, 1958).

[3] P. R. Holland, The Quantum Theory of Motion: An Account of the de Broglie-Bohm Causal Interpretation of Quantum Mechanics (Cambridge University Press, Cambridge, England, 1995).
[4] D. Bohm and B. Hiley, The de Broglie pilot wave theory and the further development of new insights arising out of it, Found. Phys. 12, 1001 (1982).

[5] B.-G. Englert, M. O. Scully, G. Süssmann, and H. Walther, Surrealistic bohm trajectories, Z. Naturforsch. A 47, 1175 (1992).

[6] D. Dürr, W. Fusseder, S. Goldstein, and N. Zanghi, Comment on "surrealistic Bohm trajectories", Z. Naturforsch. A 48, 1261 (1993).

[7] B.-G. Englert, M. O. Scully, G. Süssmann, and H. Walther, Reply to comment on "surrealistic Bohm trajectories", Z. Naturforsch. A 48, 1263 (1993).

[8] M. O. Scully, Do Bohm trajectories always provide a trustworthy physical picture of particle motion?, Phys. Scr. T76, 41 (1998).

[9] M. Golshani and O. Akhavan, Bohmian prediction about a two double-slit experiment and its disagreement with standard quantum mechanics, J. Phys. A 34, 5259 (2001).

[10] D. H. Mahler, L. Rozema, K. Fisher, L. Vermeyden, K. J. Resch, H. M. Wiseman, and A. Steinberg, Experimental nonlocal and surreal Bohmian trajectories, Sci. Adv. 2, e1501466 (2016).

[11] A. B. Nassar and S. Miret-Artés, Bohmian mechanics, Open Quantum Systems and Continuous Measurements (Springer, New York, 2017).

[12] K. G. Makris, A. Brandstötter, P. Ambichl, Z. H. Musslimani, and S. Rotter, Wave propagation through disordered media without backscattering and intensity variations, Light Sci. Appl. 6, e17035 (2017).

[13] K. G. Makris, Z. H. Musslimani, D. N. Christodoulides, and S. Rotter, Constant-intensity waves and their modulation instability in non-Hermitian potentials, Nat. Commun. 6, 7257 (2015).

[14] I. Liberal and N. Engheta, Near-zero refractive index photonics, Nat. Photonics 11, 149 (2017).

[15] See Supplemental Material at http://link.aps.org/ supplemental/10.1103/PhysRevLett.120.193902, which includes (S1) the derivation of the Bohmian representation of optical materials, (S2) the derivation of Eq. (3), (S3) non- $P T$ symmetric spatial phase trapping, and (S4) phase-conserved energy confinement and cancellation with random profiles.

[16] C. M. Bender and S. Boettcher, Real Spectra in NonHermitian Hamiltonians Having PT Symmetry, Phys. Rev. Lett. 80, 5243 (1998).

[17] S. Yu, X. Piao, J. Hong, and N. Park, Metadisorder for designer light in random systems, Sci. Adv. 2, e1501851 (2016).

[18] J. D. Joannopoulos, S. G. Johnson, J. N. Winn, and R. D. Meade, Photonic Crystals: Molding the Flow of Light (Princeton University Press, Princeton, NJ, 2011).

[19] A. Lagendijk, B. Van Tiggelen, and D. S. Wiersma, Fifty years of Anderson localization, Phys. Today 62, No. 8, 24 (2009).

[20] H. Lee, T. Chen, J. Li, K. Y. Yang, S. Jeon, O. Painter, and K. J. Vahala, Chemically etched ultrahigh-Q wedgeresonator on a silicon chip, Nat. Photonics 6, 369 (2012).

[21] D. Marinica, A. Borisov, and S. Shabanov, Bound States in the Continuum in Photonics, Phys. Rev. Lett. 100, 183902 (2008). 
[22] C. W. Hsu, B. Zhen, J. Lee, S. L. Chua, S. G. Johnson, J. D. Joannopoulos, and M. Soljacic, Observation of trapped light within the radiation continuum, Nature (London) 499, 188 (2013).

[23] N. Kirstaedter, O. G. Schmidt, N. N. Ledentsov, D. Bimberg, V. M. Ustinov, A. Y. Egorov, A. E. Zhukov, M. V. Maximov, P.S. Kop'ev, and Zh. I. Alferov, Gain and differential gain of single layer InAs/GaAs quantum dot injection lasers, Appl. Phys. Lett. 69, 1226 (1996).

[24] R. Maas, J. Parsons, N. Engheta, and A. Polman, Experimental realization of an epsilon-near-zero metamaterial at visible wavelengths, Nat. Photonics 7, 907 (2013).

[25] C. E. Rüter, K. G. Makris, R. El-Ganainy, D. N. Christodoulides, M. Segev, and D. Kip, Observation of parity-time symmetry in optics, Nat. Phys. 6, 192 (2010).

[26] L. Feng, R. El-Ganainy, and L. Ge, Non-Hermitian photonics based on parity-time symmetry, Nat. Photonics 11, 752 (2017).

[27] J. B. Pendry, D. Schurig, and D. R. Smith, Controlling electromagnetic fields, Science 312, 1780 (2006).

[28] S. Horsley, M. Artoni, and G. La Rocca, Spatial KramersKronig relations and the reflection of waves, Nat. Photonics 9, 436 (2015).
[29] M. Choi, S. H. Lee, Y. Kim, S. B. Kang, J. Shin, M. H. Kwak, K.-Y. Kang, Y.-H. Lee, N. Park, and B. Min, A terahertz metamaterial with unnaturally high refractive index, Nature (London) 470, 369 (2011).

[30] D. R. Smith, J. B. Pendry, and M. C. Wiltshire, Metamaterials and negative refractive index, Science 305, 788 (2004).

[31] L. Chang, X. Jiang, S. Hua, C. Yang, J. Wen, L. Jiang, G. Li, G. Wang, and M. Xiao, Parity-time symmetry and variable optical isolation in active-passive-coupled microresonators, Nat. Photonics 8, 524 (2014).

[32] L. Feng, Z. J. Wong, R.-M. Ma, Y. Wang, and X. Zhang, Single-mode laser by parity-time symmetry breaking, Science 346, 972 (2014).

[33] B. Peng, Ş. K. Özdemir, F. Lei, F. Monifi, M. Gianfreda, G. L. Long, S. Fan, F. Nori, C. M. Bender, and L. Yang, Parity-time-symmetric whispering-gallery microcavities, Nat. Phys. 10, 394 (2014).

[34] H. Hodaei, M.-A. Miri, M. Heinrich, D. N. Christodoulides, and M. Khajavikhan, Parity-time-symmetric microring lasers, Science 346, 975 (2014).

[35] S. Assawaworrarit, X. Yu, and S. Fan, Robust wireless power transfer using a nonlinear parity-time-symmetric circuit, Nature (London) 546, 387 (2017).

[36] S. Longhi, Quantum-optical analogies using photonic structures, Laser Photonics Rev. 3, 243 (2009). 